\title{
Mental health research priorities in low- and middle-income countries of Africa, Asia, Latin America and the Caribbean
}

P. Sharan, C. Gallo, O. Gureje, E. Lamberte, J. J. Mari, G. Mazzotti, V. Patel, L. Swartz, S. Olifson, I. Levav, A. de Francisco, S. Saxena, for the World Health Organization-Global Forum for Health Research - Mental Health Research Mapping Project Group*

\section{Background}

Studies suggest a paucity of and lack of prioritisation in mental health research from low- and middle-income (LAMI) countries.

\begin{abstract}
Aims
To investigate research priorities in mental health among researchers and other stakeholders in LAMI countries.
\end{abstract}

\section{Method}

We used a two-stage design that included identification, through literature searches and snowball technique, of researchers and stakeholders in 114 countries of Africa, Asia, Latin America and the Caribbean; and a mail survey on priorities in research.

\section{Results}

The study identified broad agreement between researchers and stakeholders and across regions regarding research priorities. Epidemiology (burden and risk factors), health systems and social science ranked highest for type of research. Depression/anxiety, substance use disorders and psychoses; and children and adolescents, women, and people exposed to violence/trauma were prioritised among the disorders and population groups respectively. Important criteria for prioritising research were burden of disease, social justice, and availability of funds. Stakeholder groups differed in the importance they gave to the personal interest of researchers as a criterion for prioritising research. Researchers' and stakeholders' priorities were consistent with burden of disease estimates, however suicide was underprioritised compared with its burden. Researchers' and stakeholders' priorities were also largely congruent with the researchers' projects.

\section{Conclusions}

The results of this first ever conducted survey of researchers and stakeholders regarding research priorities in mental health suggest that it should be possible to develop consensus at regional and international levels regarding the research agenda that is necessary to support health system objectives in LAMI countries.

\section{Declaration of interest}

P.S. has received a research grant as a principal investigator from Eli Lilly after completion of the 'Mental Health: Mapping of Research Capacity in Low- and Middle-Income Countries' project.
Health research contributes to the advancement of science, provision of solutions for health problems and to growth, development, equity, global security and the fight against poverty. ${ }^{1,2}$ Yet, health research, ${ }^{3-6}$ including mental health research, ${ }^{7-11}$ is beset with a mismatch between needs and investment (known as the 10/90 gap). In addition, it has been noted that low- and middle-income (LAMI) countries often do not address population need-based priorities. ${ }^{12,13}$ This serious gap has an adverse impact on the process of scaling up of services and programmes for mental health. ${ }^{2,14}$

Over the past two decades, explicit methods for priority setting are gradually replacing previous models that were driven by a mix of implicit as well as explicit criteria that included potential for publication in high-impact journals, financial or political interests of donors, biases of members of policy-making panels, and media exposure. ${ }^{15,16}$ Two types of explicit methods for priority setting - based on indicators of needs (e.g. burden) and values (opinion of experts/stakeholders) - have been detailed. ${ }^{16,17}$ However, composite indicators of need have gained prominence in research-priority setting because these measures (e.g. disabilityadjusted life-years) lend themselves to comparisons across a broad range of diseases and economic analyses of interventions. ${ }^{18}$ Although such indicators are useful for rational resource allocation, involvement of a wide range of stakeholders is considered essential

*Group members are listed in the Appendix. for ensuring legitimacy, transparency and fairness of priority decisions in health research investments. ${ }^{19,20}$ Participation by a broad spectrum of stakeholders helps to identify research needs, technical and financial capabilities, information gaps and extraneous political pressures, buttress the values and ethics of the society, and fosters ownership of the process and output of priority setting initiatives. Importantly, it facilitates shared responsibility and accountability in the implementation of the research agenda. ${ }^{19,21,22}$

Of late, a number of LAMI countries have conducted prioritysetting exercises in health, health systems and health policy that have successfully involved stakeholders. ${ }^{22}$ However, these have mostly involved single countries and have excluded priorities in mental health research. To fill this void in evidence, this study was designed to address the global and regional LAMI countries' priorities in mental health research.

\section{Method}

The questions included in this report were part of a larger study Mental Health: Mapping of Research Capacity in Low- and Middle-Income Countries - that aimed to develop regional maps of mental health researchers and detail their agenda and the infrastructure (e.g. institutional, funding, policy) that supports them. ${ }^{23}$ Our study is based on the section on priorities for mental health research. 
The Global Forum for Health Research (Global Forum) issued a 'Request for Proposals' that was distributed widely using electronic and postal methods to universities, research institutions and individuals in the LAMI countries of Africa, Asia, and Latin America and the Caribbean (henceforth termed the Americas). The LAMI countries of Europe and the Middle East were not included. Of 18 distinct proposals ( 2 from each region), 6 were selected based on their scope and the capabilities of the teams. With the coordination and support from the Global Forum and the World Health Organization (WHO), all project leaders met to agree on the common issues to be explored, methods and procedures, for example databases to be used, documents and networks to be tapped to identify stakeholders, shared understanding of terms and definitions. A total of 114 LAMI countries from Africa $(n=52)$, the Americas $(n=30)$ and Asia $(n=32)$ constituted the study universe (see Sharan et al for the list of countries). ${ }^{23}$

\section{Identification and survey of stakeholders}

Researchers were identified through an exhaustive search of indexed (Medline and PsycInfo) and non-indexed literature (regional databases, online journals, other local journals, unpublished papers, presentations and reports) for a 5-year period (1999-2003) for mega countries (population >100 million), and for a 10-year period (1993-2003) for less populated countries. All authors, whose addresses were identified in the literature search, were invited by mail or email to participate in this survey. Other mental health stakeholders were identified through websites and reports of organisations, journals, regional databases, grey literature searches, ministries of health documents and snowball technique. Stakeholders included: decision makers (legislators and officers of ministries of health, health insurance agencies, foundations and research councils); university administrators; and officers of associations (office bearers of associations of professionals, non-governmental organisations and associations of users and carers). Survey respondents' addresses were obtained through local directories (e.g. professional organisations), resources like Google Scholar and correspondence with affiliated institutions and colleagues.

The survey procedure was as follows: an initial letter was sent explaining the study rationale, choice in response formats (electronic or paper based) and confidentiality. One week later, the questionnaire and a pre-addressed return envelope or information about the website where the questionnaire was available was sent. Non-respondents were sent up to four reminders (with the questionnaire) at 2-4 weeks intervals. Answers could be provided in English, French, Spanish or Portuguese.

The draft questionnaire was developed by a core group (the Global Forum and WHO professionals in public mental health, health research priority setting and health economics; with work experience in LAMI countries) that compiled an initial list of items within the broad health research system framework. ${ }^{24}$ Items on priority were adapted from: an unpublished WHO questionnaire on mental health research (critiqued by 12 public mental health experts from WHO, LAMI and high-income countries); a WHO-research policy and cooperation study on health research in LAMI countries, ${ }^{3}$ and an Australian study on mental health research priorities. ${ }^{16}$ This questionnaire was subsequently critically reviewed jointly by the regional principal investigators (mental health professionals from diverse backgrounds with extensive experience of working in LAMI and high-income countries).

The section on mental health research priorities began with the question 'Over the next 5 years, what in your opinion are the most important mental health research priorities in your country?' Respondents were requested to indicate the top three for each of the following categories of research priorities by marking multiple choice boxes.

(a) Type of mental health research: epidemiological studies on the burden of disorders and risk factors; health systems research (e.g. services evaluation, policy and economic studies); social science research (e.g. illness beliefs, measurement); clinical trials; and basic sciences research (e.g. genetics, neuroimaging).

(b) Mental disorders/conditions: depression/anxiety, substance use disorders, psychoses, disorders with onset in childhood and adolescence, suicide, dementia, personality issues, learning disorders, epilepsy, eating disorders, others.

(e) Specific populations: children and adolescents, women, people exposed to violence/trauma, poor people, elderly people, disabled people, minorities, refugees, prisoners, others.

(f) Criteria for prioritising: burden of disease in the population, availability of funds, researchers' personal interests, policymaker request, social justice/equity, others.

\section{Comparison of identified priorities with external indices}

The research priorities of researchers and stakeholders were compared with two external (hard) indices that were available for the LAMI countries of Africa, Asia and the Americas: projects conducted by responding researchers ${ }^{23}$ and the burden of neuropsychiatric diseases. ${ }^{25}$ As a part of the larger study cited above, each researcher had to tick multiple choice boxes (with the same response options as in the survey on priorities) regarding the type of research, its focus on disorders and specific populations, and motivation(s) for three research projects conducted during the preceding 5 years $\left(n=1847\right.$ projects). ${ }^{23}$ A table on estimated total disability-adjusted life-years by cause and countries (December 2004 estimates) was used to compile the burden of six relevant neuropsychiatric categories for 111 of the 114 countries: depression/anxiety (unipolar depressive disorders, post-traumatic stress disorder, obsessive-compulsive disorder, panic disorder), substance use disorders (alcohol use disorders, drug use disorders), suicide (self-inflicted injury), psychosis (schizophrenia), dementia (Alzheimer and other dementias) and epilepsy. ${ }^{25}$

The study was approved by the institutional review boards of the respective teams. No formal ethical approval was required in the Philippines because the official and formal ethical board was not in existence during the conduct of the study. Formal ethical approval was also not required in Nigeria at the time of the study.

\section{Results}

Almost a third of the 4633 identified mental health researchers resided in China, India and Brazil. No mental health researcher was identified in 31 of the $114(27.2 \%)$ countries and fewer than six researchers were identified in another 26 countries $(22.8 \%)$. The overall response rate was $21.1 \%$ (Africa $34.2 \%$, Asia $13.3 \%$, the Americas 31\%); responses were received from researchers residing in 53 countries $(46.5 \%$ of 114 countries and $71.9 \%$ of countries with more than five identified researchers). The largest number of responses was received from Brazil $(n=227)$ and India $(n=125)$. Three-fifths of respondents were male. The average age of respondents was 45.4 years (s.d. $=9.5$ ). All major disciplines of mental health (psychiatry $47 \%$, nursing $18.3 \%$, psychology $12.4 \%$, social sciences $8.6 \%$, neurology and other medical disciplines $12.4 \%$, public health $3.9 \%$ ) and institutional affiliations (government/ministry $56.7 \%$, private sector $30 \%$, universities $22.3 \%$, research organisations $12.2 \%$, non-government sector $7.3 \%$ ) were represented (multiple responses were permitted). 
A total of 3829 stakeholders were identified (decision makers $22 \%$, association officers $49 \%$, university administrators $29 \%$ ). The overall response rate for the stakeholder group was $10.1 \%$ (Africa $12.8 \%$, Asia 8.9\%, the Americas 12\%). Decision makers from 31 countries $(27.2 \%)$, university administrators from 24 countries $(21.1 \%)$ and association officers from 37 countries $(32.5 \%)$ responded. No stakeholder was identified in $22(19.3 \%)$ countries. Stakeholders from the following countries formed more than $10 \%$ of the total pool of respondents in each stakeholder group: decision makers - Brazil (17.1\%) and Peru (10.5\%); university administrators - Brazil (12\%) and Colombia (12\%); and association officers - Philippines (18\%) and India (17\%).

Tables 1-4 show the percentage of the sample of researchers and stakeholders that rated the top three priority options. In addition, each table shows the percentage of research projects (conducted by the responding researchers) that were related to the same response categories as the subjective priorities.

\section{Comparison between stated priorities of researchers and stakeholders}

At the global level, researchers and stakeholders were consistent in their ranking of research priorities by type of research. They considered epidemiological studies of burden and risk factors the most important type, followed by health systems research, social science research, clinical trials and basic science research (Table 1). A two-position (or greater) difference in ranks between researchers' and stakeholders' opinion was observed for 1 of 15 intra-regional (in Africa) and 2 out of 30 inter-regional (both between stakeholders) comparisons.

The ranking of research priorities in terms of mental disorders was largely consistent across the researcher and stakeholder groups globally (Table 2). Depression/anxiety, substance use disorders, psychosis, and disorders with onset in childhood and adolescence held the first four positions. A two-position (or greater) difference in ranks was observed for two conditions at the global level; 7 of 30 intra-regional (3 each in Africa and the Americas, and 1 in Asia) and 17 of 60 inter-regional comparisons (7 for researchers and 10 for stakeholders). None of these differences included the top four ranked conditions. Large intra-regional differences (three-position or greater) between researchers and stakeholders were noted for suicide (researchers gave it a higher rank in Africa and stakeholders gave it a higher rank in the Americas) and dementia (researchers gave it a higher rank in the Americas; however, the percentage difference between the groups was small). Large inter-regional differences were noted for suicide (researchers in the Americas and stakeholders in Africa gave it a low rank), dementia (researchers in Africa and stakeholders in the Americas gave it a low rank), personality issues (stakeholders in Asia gave it a low rank) and epilepsy (stakeholders in Africa gave it a high rank).

As Table 3 indicates, researcher and stakeholder groups were again largely consistent in their ranking of research priorities in terms of specific populations at the global level. Children and adolescents, women, people exposed to violence/trauma, poor people and elderly people were considered the top five priorities. A two-position (or greater) difference in ranks was observed for 6 of 27 intra-regional ( 1 in Africa; 2 in Asia; and 3 in the Americas) and 20 of 54 inter-regional comparisons ( 6 for researchers and 14 for stakeholders). Large intra-regional differences (three-point or more) in ranks between researchers and stakeholders were noted for people affected by violence/trauma in Asia (stakeholders gave it a higher rank). Large inter-regional differences were noted for people affected by violence/trauma (researchers in Asia gave it a low rank) and disability (stakeholders in the Americas gave it a low rank).
Finally, four of the five criteria for prioritising mental health research were ranked in a similar order by researchers and stakeholders globally: burden of disease, social justice, availability of funds and specific requests made by policy makers (Table 4). A two-position (or greater) difference in ranks was observed for 5 of 15 intra-regional comparisons (2 each in Africa and Asia, and 1 in the Americas) but none of the 30 inter-regional comparisons. Researchers and stakeholders differed markedly in the rank accorded to researchers' personal interest at the global level (researchers: second rank; stakeholders: fifth rank) and in each region.

\section{Comparison with external indices}

\section{Research projects conducted by researchers}

At the global level, a two-position (or greater) difference in ranks between researchers' priorities and research projects, and stakeholders' priorities and research projects was observed for 2 of the 10 comparisons for types of research ( 1 for researchers, 1 for stakeholders); 7 of 20 comparisons for disorders (3 for researchers, 4 for stakeholders); 10 of 18 comparisons for specific populations ( 5 each for researchers and stakeholders); and 1 of 8 comparisons for criteria for prioritising research (1 for stakeholders) (Tables 1-4). A two-position difference in ranks was not observed for the disorders and specific populations that received high (first three) or low (last two) ranks. A large (three-position or greater) difference in ranks was observed for dementia (lower rank in stakeholders' opinion in comparison with research projects), personality issues (higher rank in researchers' opinion in comparison with research projects) and learning disorders (higher rank in researchers' opinion in comparison with research projects) (Table 2), people affected by poverty (higher rank in researchers' and stakeholders' opinions in comparison with research projects) (Table 3), and personal interest of researchers as a criterion for prioritising research (lower rank in stakeholders' opinion in comparison with research projects) (Table 4).

Intra-regional comparisons showed a two-point position (or greater) difference in ranks for 3 of 30 comparisons for types of research ( 2 for researchers, 1 for stakeholders), 13 of 60 comparisons for disorders (6 for researchers, 7 for stakeholders), 24 of 54 comparisons for specific populations (11 for researchers, 13 for stakeholders), and 6 of 24 comparisons for criteria for prioritising research ( 1 for researchers and 5 for stakeholders) (Table 1-4). A three-position (or greater) difference in ranks compared with projects was observed for research on health systems in the Americas (higher rank in researchers' and stakeholders' opinion compared with research projects). A three-position (or greater) difference in ranks in comparison with projects was also observed for research on suicide in Africa (lower rank in stakeholders' opinion compared with research projects); personality issues in Asia (lower rank in stakeholders' opinion compared with research projects); and suicide (lower rank in researchers' opinion compared with research projects), dementia (lower rank in stakeholders' opinion compared with research projects) and learning disorders (higher rank in researchers' and stakeholders' opinion compared with research projects) in the Americas (Table 2).

A three-position (or greater) difference in ranks was also observed for research on people affected by poverty (higher rank in researchers' and stakeholders' opinion compared with research projects) and minorities (lower rank in researchers' and stakeholders' opinion compared with research projects) in Africa; people affected by violence/trauma (higher rank in stakeholders' opinion compared with research projects), people affected by 

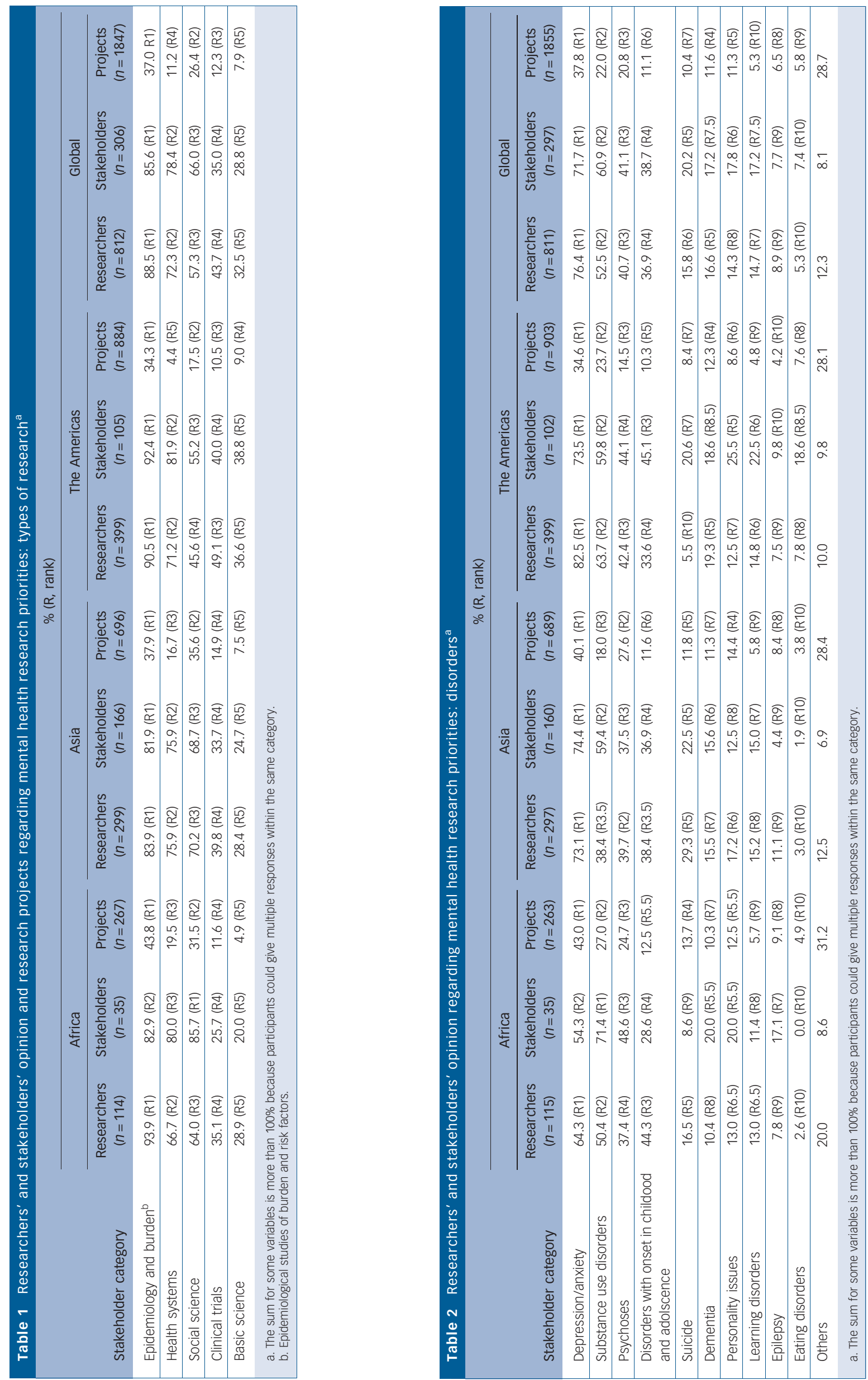

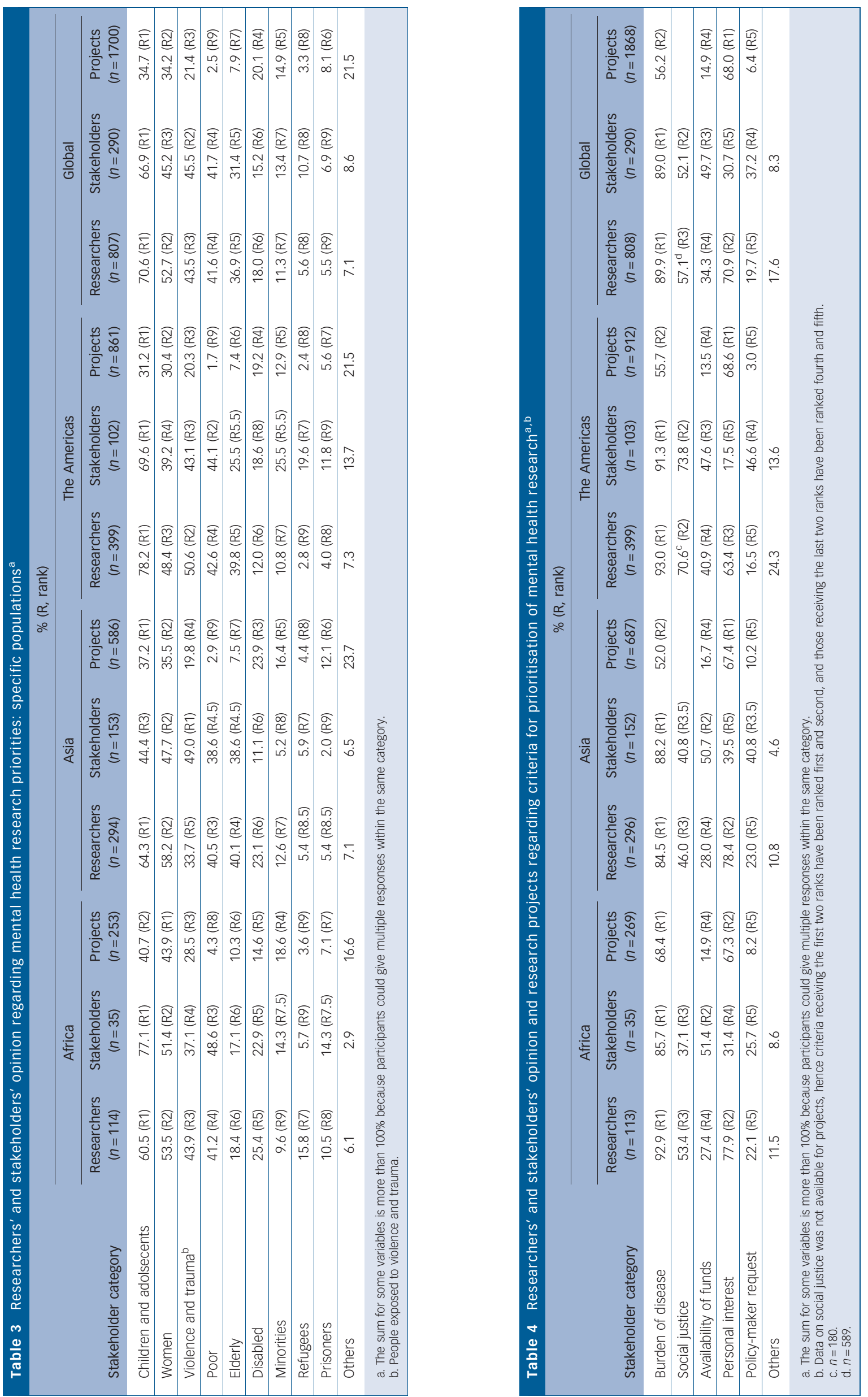


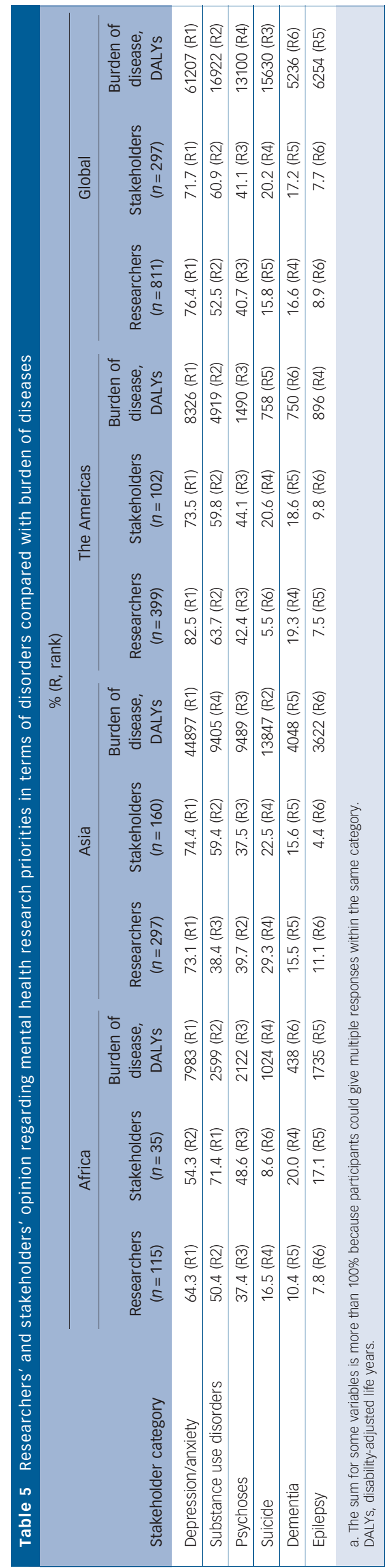

poverty (higher rank in researchers' and stakeholders' opinion compared with research projects), elderly people (higher rank in researchers' opinion compared with research projects), disabled people (lower rank in researchers' and stakeholders' opinion compared with research projects), minorities (lower rank in stakeholders' opinion compared with research projects), and prisoners (lower rank in stakeholders' opinion compared with research projects) in Asia; and people affected by poverty (higher rank in researchers' and stakeholders' opinion compared with research projects) and disabled people (lower rank in stakeholders' opinion compared with research projects) in the Americas. A difference of similar magnitude was also observed for personal interest of researchers as a criterion for prioritising research (lower rank in stakeholders' opinion compared with research projects) in Asia and the Americas.

\section{Burden of neuropsychiatric diseases}

A comparison of researchers' and stakeholders' priorities with burden of disease estimates showed broad similarities in ranking (Table 5). At the global level, a two-position difference in ranking was seen for suicide and dementia with the researchers ranking the former lower and the latter higher than their respective ranks according to burden of disease estimates. However, the per cent difference between researchers and stakeholders was small in the case of dementia. A two-position difference in ranks was observed for 8 of 36 comparisons at the intra-regional level.

\section{Discussion}

\section{Global mental health research priorities}

The study revealed broad agreement between researchers and stakeholders, and across regions regarding the priorities for mental health research in LAMI countries. These were: epidemiological studies of burden and risk factors, health systems research and social science research. The three priority mental disorders/ conditions were depression/anxiety, substance use disorders and psychoses, and the three population groups that were prioritised were children and adolescents, women and people exposed to violence/trauma. The three highest ranked criteria for prioritising research were burden of disease, social justice and availability of funds. The similarities found in priorities between researchers and stakeholders, and across regions raise genuine hopes of making research an instrument for change via collaboration among LAMI countries, researchers and stakeholders.

Researchers and stakeholders accorded epidemiological studies of burden, risk factors and health systems research the highest ranks on types of research needed. Of the 12 research options that received the highest priority scores from the Lancet Global Mental Health Group, 8 addressed health policy and systems research involving existing interventions and epidemiological research to inform priority setting. ${ }^{14}$

Depression and anxiety, which cause the greatest burden among neuropsychiatric diseases, ${ }^{25}$ were considered a priority condition by a large proportion of researchers and stakeholders. A nationwide survey of stakeholder perspectives on research priorities in Australia also showed that all stakeholder groups prioritise affective disorders. ${ }^{16}$ The prioritisation of substance use disorders and psychosis by a large proportion of researchers and stakeholders also follows the burden of disease estimates. ${ }^{25}$ In keeping with their burden, the Lancet Global Mental Health Group focused on four groups of disorders when setting priorities for global mental health research: depressive, anxiety and other common mental disorders; alcohol and other substance use disorders; child and adolescent mental disorders; and schizophrenia and other psychotic disorders. ${ }^{14}$ Compared with their burden, suicide was underprioritised and 
dementia was given greater priority in comparison with epilepsy and suicide by researchers. Suicide appeared to be similarly underprioritised in a previous audit of mental health research publications from LAMI countries. ${ }^{13,16}$ It is possible that the priority given to dementia may reflect its cost and burden of care. Also, some differences in prioritisation between the burden of disease estimates and the present study may be related to slightly different grouping of conditions used in the two data-sets, e.g. self-inflicted injuries is broader than suicide.

Despite differences in definition of population subgroups, children and adolescents and the socially and economically disadvantaged were consistently rated highly by all stakeholder groups in the Australian survey on stakeholder perspectives. ${ }^{16}$ The prioritisation of women in this study may reflect their greater risk for the highly prevalent and burdensome depressive and anxiety disorders, ${ }^{25,26}$ as well as their relatively disadvantaged status. Many international organisations have stressed the need for gender-mainstreaming in research. ${ }^{3,21}$

In the present study, a salient difference between researchers and stakeholders emerged in the importance accorded to researchers' personal interest as a criterion for priority setting. Experts in research governance suggest that ideological emphasis on 'scientific autonomy' in the process of agenda setting has been largely responsible for the lack of public health and operational emphasis in research agenda; and support the use of a systematic and transparent process of priority setting to ensure that the voice and will of the different stakeholders are respected. ${ }^{1,3,14-21}$ The higher ranking of burden of disease compared with social justice as a criterion for prioritisation of research in our study is reminiscent of the greater emphasis on disease burden in comparison with equity among LAMI country research projects on health policy and systems issues. ${ }^{27}$

Researchers' and stakeholders' ranking of subjective priorities were similar to the ranks achieved by similar response categories in projects conducted by researchers, particularly for types of research and criteria for prioritisation. A greater number of differences were noted for disorders and specific populations (mainly for medium priority disorders and sub-populations), suggesting areas that may need harmonisation through better research governance. Both researchers and stakeholders gave a much higher priority to people affected by poverty in comparison to the rank achieved by this sub-population in research projects, suggesting that research on equity needs to be given greater prominence. Indeed, Harrison has argued for the need to consider equity as a fourth research and development instrument (in addition to discovery-oriented research, innovation research, and implementation research) to ensure that research portfolios respond to the poor and the underlying health problems in LAMI countries. ${ }^{28}$ On the other hand, both researchers and stakeholders gave a much lower priority to prisoners in comparison to the number of projects done on this sub-population, suggesting that the 'captive' nature of this sub-population makes it easier to conduct research. It may be important to look into the ethical aspects of the research conducted on prisoners.

To contextualise issues related to delineating priorities for mental health research in LAMI countries, it is important to remain cognisant of the many barriers to research in poorly resourced settings with scarce financial, human and technical resources. ${ }^{29,30}$ Although data on the number of mental health researchers and research funding in LAMI countries are not readily available, the ratio of scientists per 1000 population in LAMI countries is under 0.5 , in contrast to high-income countries where the ratio is over 2. Similarly, LAMI countries invest less than $1 \%$ of their gross domestic product in research and development compared with $2 \%$ in high-income countries. ${ }^{31}$ Thus, in addition to a major revision of priorities in mental health research, efforts would be needed to increase resources (e.g. capacity and funding) available for conducting such research. ${ }^{14,32}$

Admittedly, this study did not address issues relating to the relative priority to be accorded to research in relation to other activities (e.g. services) that are needed in LAMI countries. However, influential expert groups envision an active role for research in the multidimensional efforts required to change the current mental health situation in these countries. ${ }^{2,14}$ Although, the ability of national institutions in LAMI countries to produce and use high-quality health research that is appropriate to their needs can be weak at various stages of the policy process, scientific research informs mechanisms (e.g. media, powerful advocates) that influence policy and public health. ${ }^{33,34}$ There are some good examples, where local research findings were interpreted and utilised against a background of global evidence and experience from different settings to change government policies in the mental health field in LAMI countries. ${ }^{35,36}$

\section{Regional issues}

Assessed in terms of ranks, the concordance between the subjective priorities of researchers and stakeholders was high; and even greater than the concordance between researchers' subjective priorities and the ranks given to similar response categories in research projects. Consistently, higher prioritisation of certain issues (e.g. health systems research and learning disorders in the Americas) by both researchers and stakeholders compared with the rank achieved by these in research projects are important for regional research governance.

Although there was overall similarity in the appreciation of need (burden of disorder) among researchers and stakeholders, some differences in values and interest were also evident. American stakeholders gave a lower priority to dementia compared with researchers and research projects. A similar finding was reported in the Australian survey on stakeholder perspectives, and the authors suggested that stakeholders may have a lesser appreciation of the burden caused by dementia; ${ }^{16}$ however, a discordance between researchers and stakeholders was not seen in Africa and Asia. Similarly, African stakeholders gave a lower ranking to suicide in comparison with researchers and research projects; however, researchers in the Americas gave a lower priority to the same condition compared with stakeholders. More research is required to understand the reason for the differences between researchers and stakeholders and between regions, and awareness of these differences is necessary for the process of translation of research findings into information related to regional research governance including decisions on funding. ${ }^{37}$

There were greater inter-regional differences regarding priorities between stakeholders and researchers, probably because of differences in appreciation of financial capabilities, information gaps and distortions, the political environment and the values and ethics of a given society. Hence, eliciting stakeholders' input would appear to be very relevant at each level (local, national, regional, global) of priority setting.

Larger inter-regional differences in ranking were noted for disorders given lower ranks and for many specific populations, suggesting areas where elicitation of stakeholder perspectives would be crucial - conditions for which evidence of need (e.g. burden) are lacking or differences in need are less salient and issues that appear to be particularly imbued with social values in the given context(s). Significantly, there was no inter-regional variation in the criteria for prioritisation of research, which is welcome information for consensus building and working on global priorities. 


\section{Methodological issues}

An innovative aspect of the methodology applied here was to reach out to a large and reasonably representative group of active researchers in LAMI countries by following a two-stage design, starting with mapping of researchers and stakeholders through both literature searches and snowball technique, and then surveying them through six regional teams. The large number of researchers identified in Brazil, China and India was in keeping with their population size and recognised research capacity. ${ }^{7,13}$ However, the number of responses was not proportionate to the number of identified researchers and stakeholders in each country. As in the Australian survey of stakeholder priorities, ${ }^{16}$ relevant stakeholders were more difficult to sample because there were few organised lists and it was difficult for research groups (based outside the country) to identify stakeholders who were familiar with mental health research.

Postal questionnaires are widely used to collect data in health research and appeared to be the only financially viable option for collecting information from the large, geographically dispersed populations addressed in this study. The study suffered from the disadvantage of this procedure, i.e. a low response rate that compromised representativeness, despite our intensive efforts to reduce it (use of a short questionnaire, personalised letters, pre-contact, follow-up contact and academic origin of the investigation). ${ }^{38,39}$ The low response rate could be partly explained by myriad pragmatic difficulties, e.g. difficulties in locating current contact details as a result of the mobility of researchers; their dispersion across a range of departments; poor internet facilities and telephone and postal services; and language barriers. However, it is also possible that issues related to saliency of the questionnaire for the intended participants played a role in the low response rate. Individuals might have doubted the usefulness of the questionnaire on methodological grounds (use of forced choice format to answer complex questions on research priorities) or in terms of its ability to meet its intended outcome (change in research priorities at the national/regional/global level). ${ }^{38-40}$ However, the intensity and breadth of the exercise in identifying and surveying researchers and stakeholders provides a more representative opinion on the research priorities in mental health in LAMI countries in comparison with the opinion of a few highly selected experts that form a part of most priority-setting efforts. This is a significant contribution of the study, particularly because there is no previously published data on this issue.

The convergence of values in the present study should be a strong reason for paying attention to its findings; however, it is possible that the results may have been influenced by our utilisation of a ranking methodology with prefixed options, particularly for questions with few response options (e.g. types of research and criteria for prioritisation). Stakeholders' values can be directly elicited using more sophisticated quantitative (e.g. surveys where respondents weight or rate their values) as well as qualitative (e.g. individual interviews, Delphi technique, group discussions, concept mapping) methods. ${ }^{19}$ Previous transnational studies on research priorities have utilised discussion groups ${ }^{41}$ and content analysis of projects; ${ }^{27}$ however, these studies were conducted on much fewer respondents or projects in comparison with the present survey. The nationwide stakeholder survey on research priorities in mental health in Australia utilised a methodology that was similar to our survey. ${ }^{16}$ It is also important to recognise that the higher prioritisation of some types of research, disorders and specific populations mandated by the forced choice format necessarily led to lower prioritisation of others such as clinical trials; suicide, learning disabilities and dementia; and refugees, minorities, prisoners, and those with disability. Hopefully, the lower rank achieved by the latter in the priority list would not be taken as a justification for completely neglecting efforts to research these major areas and subgroups, as only moderate-to-high priority areas (based on expert consensus) were listed in the survey. Some of the issues given relatively low priority by researchers and stakeholders may be important to research based on other yardsticks that were not addressed in our survey such as high cost of care (e.g. dementia) or potential for reduction of disease burden (e.g. clinical trials). ${ }^{42}$

The following study limitations should also be kept in mind when interpreting its results. Identified options that were outlined in the questionnaire were not compiled through an objective and repeatable method, but rather through consensus reached by panels of experts, and the decisions could be seen as driven by research experts' interest biases. Stakeholders may have less knowledge about research opportunities in mental health compared with researchers, and some stakeholders might have had difficulty in distinguishing between priorities for research and service provision. ${ }^{16}$ It is possible that disaggregating the stakeholder groups (e.g. service providers, carers) might have yielded different results. In the Australian survey, various stakeholder groups had differing perspectives on research priorities and major differences were observed between committees that evaluate research grants, and consumers and carers groups. ${ }^{16}$ Also, the questionnaire distributed to researchers in one subregion in the Americas did not include the item on social justice as a criterion for prioritising mental health research, making conclusions on this item tentative.

Finally, it is possible that researchers had selectively reported projects that matched their subjective priorities. To check on this possibility, we compared the ranking of various response categories in the projects with the ranking of similar categories in indexed publications (Pubmed and PsycInfo, $n=2397$ ) from the same countries in one region (the Americas). ${ }^{23}$ The ranking for types of research, disorders and specific populations was found to be identical (data available from the authors on request) suggesting that researchers had been reasonably objective in reporting on their projects.

\section{Implications}

The present study provides initial data regarding researcher and stakeholder priorities for mental health research in LAMI countries. Importantly, the study elicits priorities from a large pool of LAMI country stakeholders, rather than experts who often speak on their behalf. The study yielded a highly unexpected primary finding of a broad agreement regarding mental health research priorities between researchers and stakeholders and across regions; and between these priorities and disease burden and researchers' projects. Notwithstanding the fact that some of the methodological limitations of the study (e.g. responders could be considered as interested 'volunteers', use of ranking methodology with few prefixed options) might have enhanced agreement between various groups, this finding was unexpected because LAMI country stakeholders often work in relative isolation because of low numbers; geographic, economic, technological and language barriers; and also because stakeholder surveys usually yield greater differences rather than similarities. ${ }^{16}$ The data also highlighted that suicide is underprioritised compared with its relative burden and that there is a recognition of, but a lack of research on poverty. Stakeholder groups differed in the importance they gave to the personal interest of researchers as a criterion for prioritising research, a finding that underlines the utility of multiple stakeholder perspective in developing balanced priorities. 
As a result of its methodological limitations the study cannot be a definitive guide to the development of research policy; however, we believe that our results are sufficiently valid to be used as a starting point for further enquiry and development. The broad agreement between researchers and stakeholders in LAMI countries and across regions regarding research priorities in mental health is highly encouraging, and provides a framework within which to construct a consensual research agenda (that allows for critical differences in research portfolios) and suggests that mental health research can be built on solid partnerships. Coordination of various influences shaping the research portfolio can increase the impact of research on equity and can contribute to its strategic role for development. Such coordination would require interfaces and mechanisms such as research forums, which could also discuss the most desirable balance of influences.

P. Sharan, PhD, Department of Psychiatry, All India Institute of Medical Sciences, New Delhi, India; C. Gallo, MSc, Departamento de Bioquímica, Biología Molecular y Farmacología, and Laboratorios de Investigación y Desarrollo, Facultad de Ciencias y Filosofía, Universidad Peruana Cayetano Heredia, Lima, Peru; o. Gureje, DSC, Department of Psychiatry, University of Ibadan, Nigeria, Africa; E. Lamberte, PhD, Social Development Research Center, De La Salle University, Manila, Philippines; J. J. Mari, PhD, Department of Psychiatry, Federal University of São Paulo, Brazil; G. Mazzotti, PhD, Facultad de Ciencias y Filosofía and Facultad de Medicina, Universidad Peruana Cayetano Heredia, Lima, Peru; V. Patel, PhD, London School of Hygiene \& Tropical Medicine, UK, and Sangath, India; L. Swartz, PhD, Department of Psychology, Stellenbosch University, and Human Sciences Research Council, Cape Town, South Africa; S. Olifson, MSc, Global Forum for Health Research, Geneva, Switzerland; I. Levav, MD, Mental Health Services, Ministry of Health, Jerusalem, Israel; A. de Francisco, MD, Global Forum for Health Research, Geneva, Switzerland s. Saxena, MD, Department of Mental Health and Substance Abuse, World Health Organization, Geneva, Switzerland

Correspondence: Pratap Sharan, Department of Psychiatry, All India Institute of Medical Sciences, New Delhi - 110029, India. Email: pratapsharan@yahoo.com

First received 23 Jan 2008, final revision 23 Jan 2009, accepted 5 Feb 2009

\section{Funding}

This project was supported financially by the Global Forum for Health Research and the World Bank through its grant facility to the Global Forum for Health Research, and was implemented under the overall coordination and technical guidance of the Global Forum for Health Research and World Health Organization, Department of Mental Health and Substance Abuse, Evidence and Research Team. The Global Forum for Health Research was involved in study design; in the collection, analysis, and interpretation of data; in the writing of the report; and in the decision to submit the paper for publication. Neithe World Bank nor any other sponsor played any part in the design of the study, in data collection, in data analysis or interpretation, in manuscript preparation or in authorisation for publication. The views and opinions expressed in this report are those of the authors and should not be construed to represent the views of any of the sponsoring agencies or governments.

\section{Acknowledgements}

This publication is dedicated to the memory of Dr Guido Mazzotti (1961-2005), our dea colleague and friend, who worked until the end - with dedication and enthusiasm towards the success of this project, following his deep life-commitment to mental health research. Dr Guido Mazzotti acted as a member of the Group and principal investigator of the Peruvian team until 1 August 2005

We wish to thank the 914 mental health researchers and 290 stakeholders who participated in the study, taking time out of their very busy schedules to fill out the questionnaire. We also thank Juan Antonio Gálvez-Buccollini, Bernardo Guimas, Phabiola Herrera, Carlos Ordóñez, Fabián Otárola and José Carlos San Martín for writing the Herrera, Carlos Ordóñez, Fabián Otárola and José Carlos San Martín for writing the
proposal to the Global Forum for Health Research and establishing contacts. The contact people for data collection were: Sergio Rojtenberg (Argentina), Fernando Garitano-Zavala and Nils Noya (Bolivia), Jorge Téllez (Colombia), Henriette Raventos (Costa Rica), Carlos León Andrade (Ecuador), Américo Reyes (Honduras), Carmen Lara (Mexico), Isabel Riaño (Panama), César Mella (Dominican Republic), Stan Kutcher and Sonia Chehil (Canada for St. Kitts \& Nevis and Grenada) and Edgard Belfort (Venezuela). We are grateful for their assistance as well as for that of Ursula Ochoa and Rosario Vidal (data entry and crosschecking), Rocio and Cristy Crivillero (Google searches), Patricia Ferrer and Gladys Torres (coordinating contact with researchers), and Cesar Cárcamo and Jesús Castagnetto (dat handling and analysis). For their support, we thank Renato Alarcón, Sergio Aguilar-Gaxiola, Duncan Pedersen and Alejandro Llanos as well as Olawoye Fadahunsi, Olubunmi Salako and Alaba Oyekan (Nigeria); Kishori Mandrekar (India); Sisira Sirribadanna (Sri Lanka); Haider Naqvi (Pakistan); Nirupama Basnet (Nepal), and Wang Xiangdong, Bernardo Conde and Ma. Rosanna de Guzman (Philippines). Last but not least, we thank Anthony Burns for his technical assistance with database construction and Lizette Phillips for her administrative assistance (South Africa)

\section{Appendix}

\section{Members of the WHO-Global Forum for Health Research - Mental Health Research Mapping Project Group}

Brazil: Jair de Jesus Mari (Principal Investigator) and Denise Razzouk, Department of Psychiatry, Federal University of São Paulo; Jeronimo Gerolin, Centre of Evaluation and Data Integration, CAIDI, Federal University of São Paulo; Maria Thereza Dubugras, Department of Psychiatry, Federal University of São Paulo.

India: Vikram Patel (Principal Investigator), London School of Hygiene \& Tropical Medicine, UK and Sangath, India; Smita Naik, Sangath, India; Omar Rahman, Independent University, Bangladesh; Murad Khan, Aga Khan University, Pakistan; Suraj Thapa, Centre for Public Health Research and Services, Nepal; Athula Sumathipala, Forum for Research and Development, Sri Lanka.

Nigeria: Oye Gureje (Principal Investigator) and Lola Kola, Department of Psychiatry, University of Ibadan.

Peru: Guido Mazzotti (Principal Investigator of the Peruvian team until he passed away on 1 August 2005), Facultad de Ciencias y Filosofía y Facultad de Medicina; Carla Gallo (Principal Investigator), Fabián Fiestas and Giovanni Poletti, Facultad de Ciencias y Filosofía; Abel Sagástegui and Silvana Sarabia, Facultad deMedicina; Inés Bustamante, Facultad de Salud Pública y Administración,Universidad Peruana Cayetano Heredia. Philippines: Exaltacion E. Lamberte (Principal Investigator), Avelita V. Lapitan and Cecille C. Pascasio, Social Development Research Centre, De La Salle University.

South Africa: Leslie Swartz (Principal Investigator), Department of Psychology, Stellenbosch University, and Human Sciences Research Council; Kathleen McDougall, Alison Breen, Carol Legg and Margie Schneider, Human Sciences Research Council.

Technical Coordination Group: Andres de Francisco and Sylvie Olifson, Global Forum for Health Research; Shekhar Saxena, World Health Organization; Pratap Sharan and Itzhak Levav, Consultants.

\section{References}

1 Commission on Health Research for Development (COHRED). Health Research: Essential Link to Equity in Development. Oxford University Press, 1990

2 World Health Organization (WHO). Mental Health Global Action Programme. WHO, 2002.

3 Global Forum for Health Research (Global Forum). The 10/90 Report on Health Research 2001-2002. Global Forum, 2002.

4 Horton R. Medical journals: evidence of bias against the diseases of poverty. Lancet 2003; 361: 712-3.

5 Anonymous. Western medical journals and the 10/90 problem. CMAJ 2004; 170: 5

6 Rochon PA, Mashari A, Cohen A, Misra A, Laxer D, Streiner DL, et al. Relation between randomized controlled trials published in leading general medical journals and the global burden of disease. CMAJ 2004; 170: 1673-7.

7 Patel V, Sumathipala A. International representation in psychiatric literature. Survey of six leading journals. Br J Psychiatry 2001; 178: 406-9.

8 Parker G, Parker K. A profile of regional psychiatry publishing: home and away. Aust N Z J Psychiatry 2002; 36: 693-6.

9 Saxena S, Paraje G, Sharan P, Karam G, Sadana R. The 10/90 divide in mental health research: trends over a 10-year period. Br J Psychiatry 2006; 188 $81-2$

10 Ipser JC, Stein DJ. Little mental disorder research in general medical journals in low- and middle-income countries. S Afr Med J 2007; 97: 110-1.

11 Patel V, Kim YR. Contribution of low- and middle-income countries to research published in leading general psychiatry journals, 2002-2004. Br J Psychiatry 2007; 190: 77-8.

12 Flisher AJ, Parry CD, Stein DJ. To what extent does South African mental health and substance abuse research address priority issues? S Afr Med 2000; 90: 378-80.

13 Saxena S, Maulik PK, Sharan P, Levav I, Saraceno B. Mental health research on low- and middle-income countries in indexed journals: a preliminary assessment. J Ment Health Policy Econ 2004; 7: 127-31. 
14 Lancet Global Mental Health Group, Chisholm D, Flisher AJ, Lund C, Patel V Saxena S, et al. Scale up services for mental disorders: a call for action. Lancet 2007; 370: 1241-52.

15 Rudan I, Gibson J, Kapiriri L, Lansang MA, Hyder AA, Lawn J, et al: Child Health and Nutrition Research Initiative (CHNRI). Setting priorities in global child health research investments: assessment of principles and practice. Croat Med J 2007; 48: 595-604.

16 Griffiths KM, Jorm AF, Christensen H, Medway J, Dear KB. Research priorities in mental health. Part 2: an evaluation of the current research effort agains stakeholders' priorities. Aust N Z J Psychiatry 2002; 36: 327-39.

17 Campbell D. Research priorities in mental health. Can J Psychiatry 1986; 31: 746-9.

18 The Working Group on Priority Setting, Council on Health Research for Development (COHRED). Priority setting for health research: lessons from developing countries. Health Policy Plan 2000; 15: 130-6.

19 Kapiriri L, Tomlinson M, Chopra M, El Arifeen S, Black RE, Rudan I; Child Health and Nutrition Research Initiative (CHNRI). Setting priorities in global child health research investments: addressing values of stakeholders. Croat Med J 2007; 48: 618-27.

20 Daniels N, Sabin JE. Setting Limits Fairly: Can We Learn to Share Medical Resources? Oxford University Press, 2002.

21 Global Forum for Health Research (Global Forum). The 10/90 Report on Health Research 2003-2004. Global Forum, 2004.

22 Nuyens $Y$. Setting priorities for health research: lessons from low- and middle-income countries. Bull World Health Organ 2007; 85: 319-21.

23 Sharan P, Levav I, Olifson S, de Fransisco A, Saxena S. Research Capacity for Mental Health in Low- and Middle-Income Countries: Results of a Mapping Project. WHO and Global Forum, 2007.

24 Sadana R, Pang T. Health research systems: a framework for the future. Bull World Health Organ 2003; 81: 159.

25 World Health Organization (WHO). Revised Global Burden of Disease (GBD) 2002 Estimates. WHO, 2002 (http://www.who.int/healthinfo/statistics/ bodgbddeathdalyestimates.xIs).

26 Patel V, Chisholm D, Kirkwood BR, Mabey D. Prioritizing health problems in women in developing countries: comparing the financial burden of reproductive tract infections, anaemia and depressive disorders in a community survey in India. Trop Med Int Health 2007; 12: 130-9.

27 Gonzalez-Block MA. Health policy and systems research agendas in developing countries. Health Res Policy Syst 2004, 2: 6.

28 Harrison D. Health research: an essential tool for achieving development through equity. In Forging Links for Health Research. Perspectives from the
Council on Health Research for Development (eds V Neufeld, N Johnson): 41-80. International Development Research Centre (Canada), 2001.

29 Saxena S, Thornicroft G, Knapp M, Whiteford H. Resources for mental health: scarcity, inequity, and inefficiency. Lancet 2007; 370: 878-89.

30 Jacob KS, Sharan P, Mirza I, Garrido-Cumbrera M, Seedat S, Mari JJ, et al. Mental health systems in countries: where are we now? Lancet 2007; 370 $1061-7$.

31 United Nations Educational, Scientific and Cultural Organization (UNESCO). UNESCO Science Report 2005. UNESCO Publishing, 2005 (http:// www.unesco.org/science/psd/publications/science_report2005.pdf)

32 Hofman K, Ryce A, Prudhomme W, Kotzin S. Reporting of non-communicable disease research in low-and-middle-income countries: a pilot bibliometric analysis. J Med Libr Assoc 2006; 94: 415-20.

33 Whiteford $\mathrm{H}$. Can research influence mental health policy? Aust $\mathrm{N} Z \mathrm{~J}$ Psychiatry 2001; 35: 428-34.

34 Asbridge M. Public place restrictions on smoking in Canada: assessing the role of the state, media, science and public health advocacy. Soc Sci Med 2004; 58: 13-24.

35 Araya $\mathrm{R}$, Rojas $\mathrm{G}$, Fritsch $\mathrm{R}$, Frank $\mathrm{R}$, Lewis $\mathrm{G}$. Inequities in mental health care after health care system reform in Chile. Am J Public Health 2006; 96 : 109-13.

36 Harpham T, Tuan T. From research evidence to policy: mental health care in Viet Nam. Bull World Health Organ 2006; 84: 664-8.

37 Hyder AA, Bloom G, Leach M, Syed SB, Peters DH, Future Health Systems: Innovations for Equity. Exploring health systems research and its influence on policy processes in low income countries. BMC Public Health 2007, 7: 309.

38 Edwards $\mathrm{P}$, Roberts I, Clarke M, DiGuiseppi C, Pratap S, Wentz R, et al. Increasing response rates to postal questionnaires: systematic review. $B M J$ 2002; 324: 1183-90

39 Nakash RA, Hutton JL, Jørstad-Stein EC, Gates S, Lamb SE. Maximising response to postal questionnaires - a systematic review of randomised trials in health research. BMC Med Res Methodol 2006; 6: 5.

40 Young J. Mail surveys of general practice physicians: response rates and non-response bias. Swiss Med Wkly 2005; 135: 187-8.

41 Lionis C, Stoffers HE, Hummers-Pradier E, Griffiths F, Rotar-Pavlic D, Rethans $\mathrm{JJ}$. Setting priorities and identifying barriers for general practice research in Europe. Results from an EGPRW meeting. Fam Pract 2004; 21: 587-93.

42 Patel V, Araya R, Chatterjee S, Chisholm D, Cohen A, De Silva M, et al. Treatment and prevention of mental disorders in low-income and middleincome countries. Lancet 2007; 370: 991-1005. 